\title{
Data Quality Evaluation of ZY-1 02C Satellite
}

\author{
Mingquan $\mathrm{Wu}^{2}$, Jie Wang ${ }^{1,}$, Ni $\mathrm{Yao}^{3}$, Zhongwei $\mathrm{Hou}^{4}$, and Changyao Wang ${ }^{2}$ \\ ${ }^{1}$ Institute of Remote Sensing and Earth Sciences, Hangzhou Normal University, \\ Hangzhou 310036, China \\ wangjie1022@163.com \\ ${ }^{2}$ The State Key Laboratory of Remote Sensing Science, Institute of Remote Sensing \\ Applications, Chinese Academy of Sciences, Beijing 100101, China \\ liexuewuwei@163.com \\ ${ }^{3}$ Beijing University of Agriculture, Beijing 102206, China \\ ${ }^{4}$ Liaoning Engineering Survey and Design Institute, Jinzhou Liaoning 121000, China
}

\begin{abstract}
ZY-1 02C satellite is a new Chinese civil remote sensing satellite launched on December 22, 2011. It is the highest resolution civil remote sensing satellite in China which carried panchromatic/multispectral sensor and high resolution sensor. Compared with image of SPOT5 satellite, the radiation quality of ZY-1 $02 \mathrm{C}$ sensors data was evaluated. The result showed: the SNR of ZY-1 $02 \mathrm{C}$ is higher than SPOT5 panchromatic band; the power spectrum of ZY-1 02C is close to SPOT5 panchromatic band; however, the mean value and variance of ZY-1 02C data are smaller than SPOT5 panchromatic band, while the skewness and kurtosis of ZY-1 02C data are higher than SPOT5 panchromatic band; the definition of ZY-1 02C data is much smaller than SPOT5 panchromatic band and the entropy is a little smaller than SPOT5 panchromatic band.
\end{abstract}

Keywords: ZY-1 02C satellite, Data quality evolution, Signal to Noise Ratio, Entropy, Definition.

\section{$1 \quad$ Introduction}

Chinese resource satellite (ZY-1 02C) is a new civil remote sensing satellite which was launched on December 22, 2011. It weighs about $2100 \mathrm{~kg}, 3$ years design life, and equipped with panchromatic/multispectral sensor (P/MS) and High-resolution sensor (HR). Its mainly usage is to acquire high-resolution panchromatic and multispectral data for land resources survey and monitoring, disaster prevention and mitigation, forestry, water conservancy, ecological environment, and the state of major projects monitoring.

Data quality evolution is not only an important technology to inspect the design of satellite sensors, but also an important theoretical reference for remote sensing satellite sensor development and band selection[1]. In the last decades, several approaches have been proposed in this study area. Yang YY et.al. [2] evaluated extended service image of CBERS-01, Wang J et.al. [3] evaluated image of CBERS-02, Zhou YB et.al. [4] and Zhang JL et.al. [5] evaluated image of CBERS-02B, Liu R et.al. [6] evaluated image of HJ-1 satellite, Zhang JL et.al. [7] evaluated image of Hyperion sensor onboard on EO1satellite, Wang QJ et.al. [8] evaluated image of LISS3 sensor onboard on IRS-P6

\footnotetext{
* Corresponding author.
} 
satellite, G H Thomson[9] evaluated image of Arkon-2 satellite. Most of those methods are based on image engineering. We would like to draw your attention to the fact that it is not possible to modify a paper in any way, once it has been published. This applies to both the printed book and the online version of the publication. Every detail, including the order of the names of the authors, should be checked before the paper is sent to the Volume Editors.

\section{Study Area}

The test area covers a surface of about $104 \mathrm{~km}^{2}$ and covered by images of SPOT5 and $\mathrm{ZY}-0102 \mathrm{C}$ satellite. It is located in the north-east of Beijing, China $\left(40.05^{\circ}-40.58^{\circ}\right.$ $\mathrm{N}, 116.51^{\circ}-117.21^{\circ} \mathrm{E}$ ) (fig. 1 ). Most of the area is flat and devoted to agriculture, with a prevalence of winter wheat, corn and urban areas.

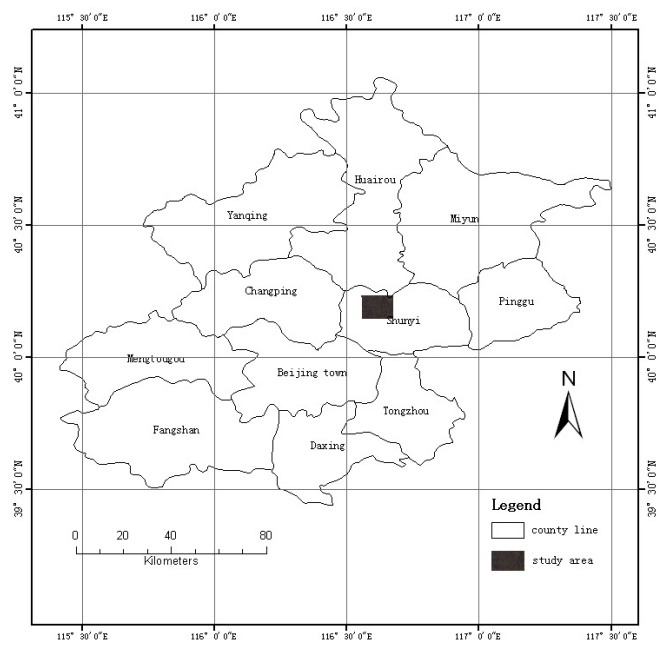

Fig. 1. Location of the study area

\section{Data Introduction}

Three ZY-1 02C images and one SPOT5 image were used in this study (Table 1). All of those data were acquired in clear shy conditions and covered the same area.

Table 1. Images used in this study

\begin{tabular}{llllll}
\hline Satellite & ZY-1 02C & & & & SPOT5 \\
\hline Sensor & 02C-MS & 02C-PAN & 02C-HR & PAN & Sensor \\
Path/row & $1 / 107$ & $1 / 107$ & $395 / 107$ & $281 / 269$ & Path/row \\
Acquisition time & $2012 / 02 / 01$ & $2012 / 02 / 01$ & $2012 / 02 / 13$ & $2007 / 04 / 24$ & Acquisition time \\
Spatial resolution & $10 \mathrm{~m}$ & $5 \mathrm{~m}$ & $2.36 \mathrm{~m}$ & $2.5 \mathrm{~m}$ & Spatial resolution \\
Sensor & 02C-MS & 02C-PAN & 02C-HR & PAN & Sensor \\
\hline
\end{tabular}




\subsection{ZY-1 02C Satellite}

ZY-1 02C is a high spatial resolution civil remote sensing satellite. It carried one panchromatic/multispectral sensor (P/MS) and two High-resolution cameras (HR) (Table 2).

Table 2. Basic parameters of sensors carried on ZY-1 02C and SPOT5 satellite

\begin{tabular}{|c|c|c|c|c|c|}
\hline Satellite & $\mathrm{ZY}-102 \mathrm{C}$ & & & SPOT5 & \\
\hline \multirow{2}{*}{ Sensor } & \multicolumn{2}{|l|}{$\mathrm{P} / \mathrm{MS}$} & \multirow{2}{*}{ HR } & \multirow{2}{*}{ PAN } & \multirow{2}{*}{ MS } \\
\hline & PAN & MS & & & \\
\hline \multirow{4}{*}{ Bandwidth $(\mu \mathrm{m})$} & \multirow{4}{*}{$0.51-0.85$} & & \multirow{4}{*}{$0.50-0.80$} & \multirow{4}{*}{$0.49-0.69$} & $0.43-0.47$ \\
\hline & & $0.52-0.59$ & & & $0.49-0.61$ \\
\hline & & $0.63-0.69$ & & & $0.61-0.68$ \\
\hline & & $0.77-0.89$ & & & $0.78-0.89$ \\
\hline Spatial resolution & $5 \mathrm{~m}$ & $10 \mathrm{~m}$ & $2.36 \mathrm{~m}$ & $2.5 \mathrm{~m}$ or $5 \mathrm{~m}$ & $10 \mathrm{~m}$ \\
\hline Swath Width & $60 \mathrm{~km}$ & & $54 \mathrm{~km}$ & $60 \mathrm{~km}$ & \\
\hline Revisit Time(day) & $3-5$ & & $3-5$ & $2-3$ & \\
\hline
\end{tabular}

\subsection{SPOT5 Satellite}

SPOT5 satellite is the fifth satellite of France SPOT series. It carried two High Resolution Geometric (HRG) sensors, one High-Resolution Stereoscopic (HRS) imaging instrument, and one Vegetation sensor.

Please note that, if your email address is given in your paper, it will also be included in the meta data of the online version.

\section{Approach}

\subsection{Qualitative Evaluation}

Image quality of satellite data can be evaluated qualitatively by visual interpretation approach or histogram comparison method. Visual interpretation method judged the image quality is good or bad directly by naked eyes, while histogram comparison method determined the image quality by histogram comparison. Images with good quality have a smoother and broader histogram.

\subsection{Quantitative Evaluation}

Radiation quality of satellite data can be evaluated quantitatively by parameters such as radiation precision, Signal to Noise Ratio, sharpness, information content, and power spectrum. 
Radiation Precision. Radiation precision is an indicator that reflects the radiation situation of images. It can be measured by parameters like mean, variance, skewness and kurtosis. The calculation formulas are as follows:

$$
\begin{gathered}
\text { Mean }=\bar{x}=\frac{1}{N} \sum_{j=0}^{N-1} x_{j} . \\
\text { Variance }=\frac{1}{N-1} \sum_{j=0}^{N-1}\left(x_{j}-\bar{x}\right)^{2} \\
\text { Skewness }=\frac{1}{N} \sum_{j=0}^{N-1}\left(\frac{x_{j}-\bar{x}}{\sqrt{\text { Variance }}}\right)^{3} . \\
\text { Kurtosis }=\frac{1}{N} \sum_{j=0}^{N-1}\left(\frac{x_{j}-\bar{x}}{\sqrt{\text { Variance }}}\right)^{4}-3
\end{gathered}
$$

Where $\mathrm{N}$ is the number of pixels, $\mathrm{j}$ is the index of pixels, and $x_{j}$ is the value of pixel $\mathrm{j}$.

Signal to Noise Ratio. Signal to Noise Ratio is the ratio of signal and noise in image. There are many different methods to calculate the image SNR, and different methods have different result. Some research showed that the image SNR and land cover closely related [10]. Variance method can determine the SNR level of sensors, but the method is influenced by surface target. However, using this method between different sensors in the same area can be used to compare the SNR level between different remote sensing sensors. The calculation formula of SNR by variance method is followed:

$$
\text { SNR }=\text { Mean } / \text { Variance } .
$$

Sharpness. The sharpness reflects the sensitivity of the image edge change. The more distinctive of color contrast of the image junction, the clearer of the image. A improved point sharpness algorithm was used to calculate the image sharpness1. The calculation formula is followed:

$$
p=\frac{\sum_{i=1}^{m \times n} \sum_{\alpha=1}^{8}|d f / d x|}{m \times n} .
$$

Where $p$ is the sharpness, $m$ and $n$ is the line and row number of pixels, $d f$ is the amplitude of intensity change, $d x$ is the distance increment between pixels. 
Information Content. Information content is a indictor that reflect the rich degree of information contained in images. It always measured by information entropy. The calculation formula of entropy is followed:

$$
H=-\sum_{i=1}^{n} P_{i} \log P_{i}
$$

Where $H$ is the information entropy, $i$ is the value of pixels, $P_{i}$ is the probability of value $i$. Due to the cross correlation between adjacent bands, the redundant information must be removed when calculating total entropy of multiband images [11].

Power Spectrum. Image power spectrum reflects the image texture features. When the image quality depredated, the loss of high frequency components will lead to blur of the image space texture and the image power spectrum of each component will fall. Thus, the comparative evaluation of image quality can be evaluated by comparing the image power spectrum12. Image power spectrum can calculate by Fourier transform. The calculation formula is followed:

$$
\text { sum }=\sum_{u=0}^{m-1} \sum_{v=0}^{n-1}|\hat{F}(u, v)|^{2} \Delta u \Delta v
$$

Where sum is image power spectrum, $u$ and $v$ is the index of pixels, $\hat{F}(u, v)$ is the value of pixel (u, v) after Fourier transform.

\section{$5 \quad$ Result and Analysis}

\subsection{Result of Qualitative Evaluation}

Through visual interpretation approach, we can found out that both SPOT5 image and ZY-1 02C images have a good visual effect (Fig.2). Land cover types like farm land, water and cities were very clear. However, those land cover types have a more strong contrast in SPOT5 image.

The histogram of SPOT5 PAN band image, and images of ZY-1 02C were calculated by ENVI 4.3 (fig.3). By comparing histogram of those images, we found out that the quality of SPOT5 PAN band image is better than ZY-1 02C images. The histogram of SPOT5 PAN band image is more broad and more smooth, while the histogram of ZY-1 $02 \mathrm{C}$ images were narrow.

\subsection{Result of Quantitative Evaluation}

Parameters of radiation precision, Signal to Noise Ratio, sharpness, information content, and power spectrum were calculated to evaluate the radiation quality of ZY-1 02C data and SPOT5 PAN band image (table 3 ). 

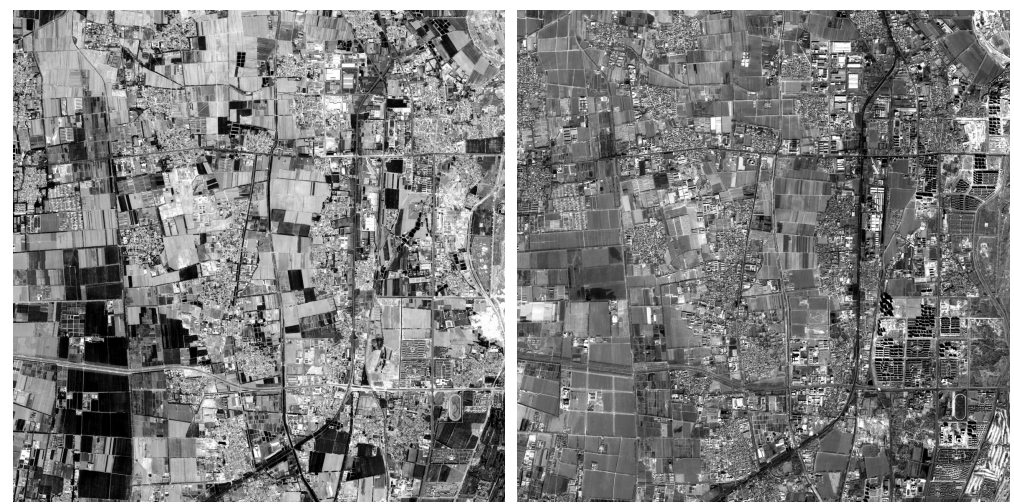

SPOT5 PAN

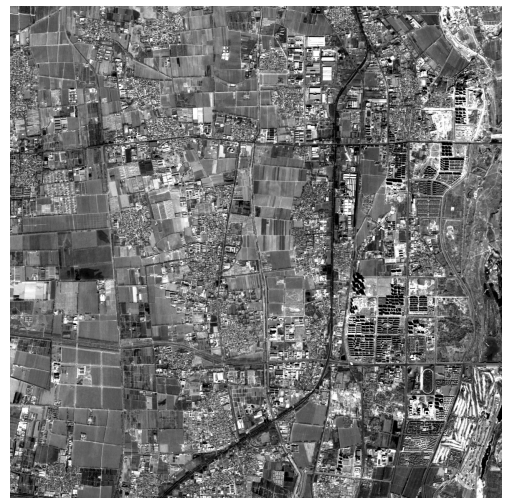

ZY-1 02C PAN

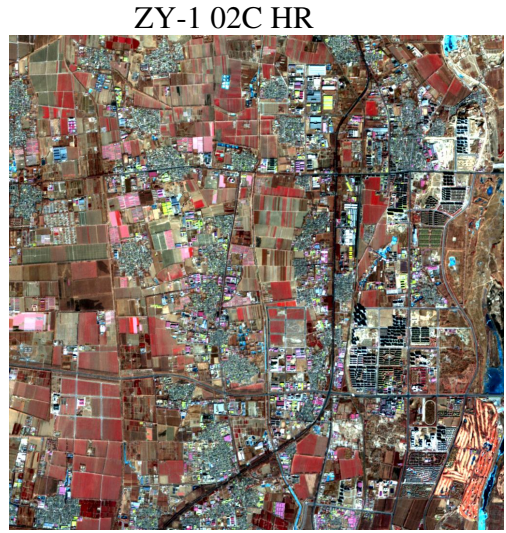

ZY-1 02C false colour image

Fig. 2. Images of ZY-102C and SPOT5
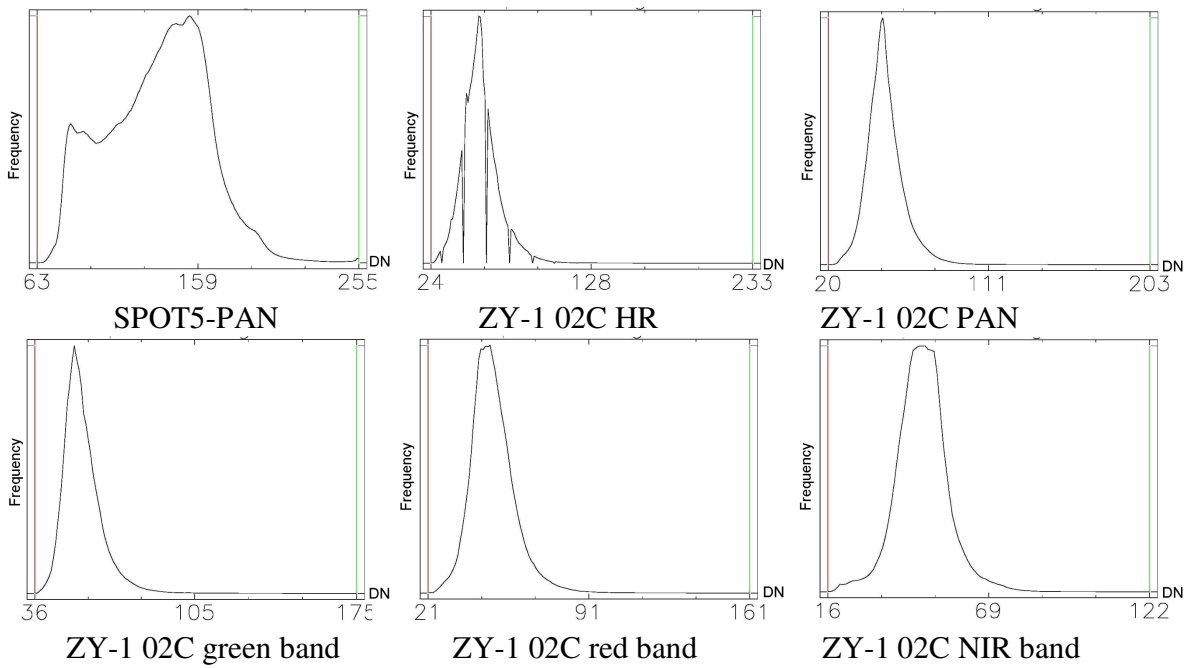

Fig. 3. Histogram comparison of different sensors 
Table 3. Quantitative evaluation results of radiation quality

\begin{tabular}{lllllll}
\hline \multirow{2}{*}{ Parameters } & \multirow{2}{*}{ SPOT } & HR & Pan & \multicolumn{2}{l}{ MUX } \\
\cline { 5 - 7 } & & & & Green & Red & NIR \\
\hline Mean & 135.917 & 57.246 & 52.990 & 56.905 & 49.918 & 47.813 \\
Variance & 882.993 & 134.722 & 101.315 & 66.393 & 85.967 & 64.759 \\
Skewness & -0.087 & 0.984 & 1.023 & 1.698 & 1.058 & 0.286 \\
Kurtosis & -0.284 & 3.449 & 3.742 & 7.058 & 3.435 & 1.866 \\
SNR & 4.574 & 4.932 & 5.264 & 6.984 & 5.384 & 5.942 \\
Sharpness & 33.115 & 16.339 & 19.899 & 17.586 & 20.800 & 17.812 \\
Power & 115.096 & 107.559 & 100.855 & 95.394 & 94.314 & 93.914 \\
spectrum & & & & & & \\
Entropy & 6.816 & 5.388 & 5.280 & 4.596 & 4.970 & 5.003 \\
Total entropy & 6.816 & 5.388 & 5.280 & 6.649 & & \\
\hline
\end{tabular}

By comparing parameters of mean, variance, skewness and kurtosis, we found that the mean and variance of SPOT5 PAN band image is higher than any ZY-1 02C images, while the skewness and kurtosis are smaller. This showed that the value distribution of SPOT5 PAN band image is more smooth and broad, which is coincide with the result of histogram comparison.

The SNR of ZY-1 02C images are higher than SPOT5 PAN band image. The green band image of ZY-1 02C MS sensor has the highest SNR. It is 53\% higher than SPOT5 PAN band image, while the red and near-infrared band is $26 \%$ and $30 \%$ higher than SPOT5 PAN band image. The SNR of pan band of ZY-1 02C is $25 \%$ higher than SPOT5 PAN band image. The HR band of ZY-1 02C has a very close SNR with SPOT5 PAN band, which is only $8 \%$ higher than SPOT5 PAN band.

The sharpness of SPOT5 PAN band is more higher than any ZY-1 02C images. The HR band of ZY-1 02C has the highest sharpness in ZY-1 02C images. However, its sharpness is only $51 \%$ of the sharpness of SPOT5 PAN band.

Images of those two satellites have a close power spectrum. The power spectrum of SPOT5 PAN band is a little higher than images of ZY-1 02C.

The SPOT5 PAN band has higher entropy than any ZY-1 02C images. The entropy of SPOT5 PAN band is about 21\%、23\%、33\%、27\% and $27 \%$ higher than the entropy of HR band, pan band, green, red and near-infrared band. Even the total entropy of MS images is also a little lower than the entropy of SPOT5 PAN band.

\section{Conclusions and Discussion}

\subsection{Conclusions}

Comparing with SPOT5 PAN band, the quality of ZY-1 02C images is similar to SPOT5 PAN band. Each data has distinct advantages and disadvantages.

The SNR of ZY-1 02C images are higher than SPOT5 PAN band. And the Power spectrum of ZY-1 02C images is close to SPOT5 PAN band. However, due to the histogram of ZY-1 02C images is too narrow and abrupt, the ZY-1 02C images cannot 
distinguish the differences of the surface reflectance very well. The sharpness of ZY-1 02C images is lower than SPOT5 PAN band. Moreover, the entropy of ZY-1 02C images are lower than SPOT5 PAN band.

The ZY-1 02C has not blue band images. This will limit the application of ZY-1 $02 \mathrm{C}$ data in water monitoring. However, ZY-1 02C have two Panchromatic bands. So the panchromatic data acquisition ability of ZY-1 $02 \mathrm{C}$ satellite is stronger than SPOT5 satellite.

\subsection{Discussion}

Due to the lack of higher-resolution topographic maps, the positioning accuracy of ZY-1 02C satellite was not evaluated in this paper. However, the positioning accuracy is a very important index of satellite image quality. So, we will carry out work in this area when conditions are ripe.

Due to data constraints, only SPOT5 PAN band was used as a comparative data. And comparison of multi-spectral bands between SPOT5 and ZY-1 02C satellite was not carried out. The difference of spatial resolution may influence the result of some comparison between SPOT5 PAN band and multispectral bands of ZY-1 02C satellite. Moreover, the acquire date of SPOT5 PAN band is five years earlier than ZY-1 02C images. The change of land cover was not considered in the evolution which has a certain extent effect to evolution result.

Acknowledgments. This work was supported by the opening foundation of Institute of Remote Sensing and Earth Sciences, Hangzhou Normal University, and the Major State Basic Research Development Program of China (2010CB950603).

\section{References}

1. Liu, R., Sun, J., Wang, J., et al.: Data quality evaluation of chinese HJ CCD sensor. Advances in Earth Science 26, 971-979 (2011)

2. Yang, Y., Wang, H., Wang, J., Xia, D.: Reseach on the quality of exceed images provided by CBERS-1 Satellite. Spacecraft Recovery and Remote Sensing 12, 34-39 (2003)

3. Wang, J., Yang, Y., Wang, H., Xu, Y., Xia, D.: The quality evaluation of the Image Date of CBERS-1No. 2 Satellite. Spacecraft Recovery and Remote Sensing 25, 34-38 (2004)

4. Zhou, Y., Tian, Q., Zhang, X.: CBERS-02B CCD image data quality evaluation and application potential for vegetation classification. Applications of Remote Sensing 6, 47$52(2008)$

5. Zhang, J., Zhao, Y., Zhang, J., et al.: The image quality evaluation of CBERS-02B and the application of the satellite of the interpretation of ore-forming factors of uranium deposits. Remote Sensing for Land and Resources 79, 69-73 (2009)

6. Yang, Z., Gu, S., Qiu, H., et al.: CBERS-1's CCD image quality evaluating and cross calibrating study. Journal of Remote Sensing 78, 113-120 (2004)

7. Zhou, Y., Tian, Q.: Image quality evaluation of EO-1 Hyperion sensor. Geo-Information Science 10, 678-683 (2008)

8. Wang, Q., Tian, Q.: Quality evaluation of LISS3 image from IRS-P6 satellite. Geography and Geo-Information Science 23, 11-14 (2007) 
9. Thomson, G.H.: Evaluation of Russian Arkon-2 Earth observation satellite. The Imaging Science Journal 53, 163-173 (2005)

10. Liu, J., Zheng, L., Tong, Q.: Estimation of signal to noise ratio of remote sensing images. Journal of Basic Science and Engineering 7, 360-365 (1999)

11. Lin, Z., Zhang, Y.: Measurement of information and uncertainty of remote sensing and GIS data. Geomatics and Information Science of Wuhan University 31, 569-572 (2006)

12. Balboa, R., Grzywacz, N.: Power spectra and distribution of contrasts of natural images from different habitats. Vision Research 43, 2527-2537 (2003) 